\title{
Interactions between dark septate endophytes, ectomycorrhizal fungi and root pathogens in vitro
}

\author{
Berthelot Charlotte $^{1}$, Corinne Leyval ${ }^{1}$, Chalot Michel $^{2,3}$, Blaudez Damien ${ }^{1 \#}$ \\ ${ }^{1}$ Université de Lorraine, CNRS, LIEC, F-54000 Nancy, France \\ ${ }^{2}$ Université de Bourgogne Franche-Comté, UMR CNRS 6249 Laboratoire Chrono- \\ environnement, F-25211, Montbéliard, France. \\ ${ }^{3}$ Université de Lorraine, F-54000 Nancy, France. \\ \# Corresponding author: \\ Dr Damien Blaudez \\ UMR 7360 LIEC, Faculté des Sciences et Technologies \\ Université de Lorraine, BP70239 \\ Vandoeuvre-lès-Nancy F-54506, France \\ Tel: +33-3-72-74-51-67 \\ E-mail: damien.blaudez@univ-lorraine.fr
}

\begin{abstract}
Dark septate endophytes (DSEs) are widely distributed worldwide and can promote plant growth. Therefore, they are considered potentially important plant allies, especially in stressful environments. Previous studies reported that DSEs cohabit roots with other microorganisms such as ectomycorrhizal (ECM), endophytic and pathogenic fungi/ oomycetes. However, interactions between different DSE species have not yet been reported, and studies on the interactions between DSEs and other fungi are scarce. Using a simple and reproducible pairwise growth assay in vitro, we studied the synergistic/antagonistic interactions between eight DSEs, two ECM fungi and three root pathogens. Most of the DSE/DSE outcomes were neutral. Interestingly, we identified several DSE strains acting in synergy with other strains, as well as strains that could potentially act as biocontrol agents. Notably, three metal-tolerant DSE strains, namely, Cadophora sp., Leptodontidium sp. and Phialophora mustea, could decrease the growth of the root phytopathogens Pythium intermedium, Phytophthora citricola and Heterobasidion annosum. The present data are discussed in the general context of the use of fungal consortia as inocula in the tree-based phytomanagement of marginal lands.
\end{abstract}

Keywords: Dark septate endophyte; ectomycorrhizal fungi; root pathogenic fungi; Leptodontidium sp.; Cadophora sp.; Phialophora mustea; Phialocephala fortinii 


\section{Introduction}

Dark septate endophytes (DSEs) are ascomycetous fungi with dark septate hyphae that are frequent root colonizers of healthy plants. DSEs may promote plant growth and fitness through the improvement of plant nutrition and stress tolerance. For instance, DSEs could increase the uptake of nitrogen and phosphorus by host plants and improve the growth, chlorophyll concentration and transpiration rate of plants (Caldwell et al. 2000; Likar and Regvar 2013). Moreover, DSEs play a key role in plant protection against abiotic (e.g., drought, salinity and pollution) and biotic stresses (e.g., plant pathogens) (Mandyam and Jumpponen 2005; Likar and Regvar 2013). DSEs are widespread among the plant kingdom; they are observed in the roots of more than 600 species spanning 100 plant families, and they have been isolated from species ranging from herbaceous to woody plants (Jumpponen and Trappe 1998). Generally, DSEs do not exhibit any host specificity. As DSEs are common in the roots of diverse plants in many environments, they usually interact with other root microorganisms. Notably, DSEs have been isolated from plants in symbiosis with arbuscular, ericoid, orchid or ectomycorrhizal (ECM) fungi (Jumpponen and Trappe 1998; Mandyam and Jumpponen 2005). Moreover, root observations related the presence of DSEs to the presence of plant growthpromoting (PGP) fungi such as mycorrhizal or endophytic fungi (Vaz et al. 2012; Massenssini et al. 2014; Op De Beeck et al. 2015). However, previous studies have almost solely focused on a given fungus and its impact on plant growth and protection against different stresses. Few studies have highlighted the possible interactions between DSEs and other root-inhabiting fungi (Reininger et al. 2012; Reininger and Sieber 2012; Della Monica et al. 2015). So far, such interactions have mostly been studied between DSEs and mycorrhizal fungi and have demonstrated effects ranging from inhibitory to synergistic, thus modifying the potential of DSEs for promoting plant growth (Cano and Bago 2005; Fryar et al. 2005; Pereira et al. 2012). As a first step towards the selection of fungal consortia suitable for plant biomass improvement and protection, it is necessary to quantify the synergistic/antagonistic relationships occurring between these fungi. In the present study, we used a simple and easily reproducible in vitro assay to conduct dual co-culture experiments between different DSEs or between DSEs and ectomycorrhizal or pathogenic fungi/oomycetes in order to test whether the outcomes of interactions between different DSE strains would be mostly neutral and those between DSEs and these other root inhabitants would be mostly antagonistic.

\section{Material and methods \\ Strains, medium and co-cultures}

Eight DSE strains (Leptodontidium sp. Me07, Leptodontidium sp. Me10, Leptodontidium sp. Pr30, Leptodontidium sp. PMI_412, Cadophora sp. Fe06, Phialocephala fortinii Pr28, Phialophora mustea $\operatorname{Pr} 27$, P. mustea Pr29) and two ectomycorrhizal strains, namely, Paxillus involutus ATCC 200175 and Hebeloma cylindrosporum D2, that were previously isolated and described (Berthelot et al. 2016) were used. The fungal pathogen Heterobasidion annosum Han 1 and two oomycetes, namely, Phytophtora citricola Cit3 and Pythium intermedium Pyint 1 came from the fungal collection of INRA Champenoux (France). The strains were grown on malt extract agar (MEA) medium (malt extract $12 \mathrm{~g} / \mathrm{l}$; agar $15 \mathrm{~g} / \mathrm{l}$ ) at $24^{\circ} \mathrm{C}$ in the dark. To pinpoint the interactions between the fungi, co-cultures were carried out in Petri dishes (diameter of $13.5 \mathrm{~cm}$ ) containing $45 \mathrm{ml}$ of MEA medium. Each plate was inoculated with two $8 \mathrm{~mm}$ diameter discs cut from 2-week-old mycelia. The fungal plugs were placed $4 \mathrm{~cm}$ from the border of the Petri dish (Fig. S1a). The control plates consisted of two plugs of the same strain. Four replicates per interaction combination were prepared; the combinations included DSE/DSE, DSE/ECM fungi or DSE/pathogen. Due to the rapid growth of the three root pathogens, the co-cultures involving these strains were carried out with the same protocol described above, except that the pathogen plugs were placed on the agar medium one week 
after the other fungi were inoculated. After two weeks of growth, the inhibition zone (IZ, defined as the distance between the two strains), the diameter, and the internal and external radii of the colonies were measured, and the ratio between the internal and external radii (IR/ER) for the interaction combinations were calculated and compared to those of the controls. The effect of the DSE strains on the diameter and the IR/ER ratio of the other strains were compared by calculating an index response for both the IR/ER ratio and for the diameter. The index responses of the IR/ER ratio (IRr) and of the diameter (IRd) were calculated with the following formula:

$$
\text { IRd of } X=\frac{\sum_{N}^{i=1} \text { effect of } X \text { on the diameter of } Y i-\sum_{N}^{i=1} \text { effect of } Y i \text { on the diameter of } X}{N}
$$

$$
I R r \text { of } X=\frac{\sum_{N}^{i=1} \text { effect of } X \text { on the ratio } I R / E R \text { of } Y i-\sum_{N}^{i=1} \text { effect of } Y i \text { on the ratio } I R / I E \text { of } X}{N}
$$

Where $\mathrm{X}$ is the strain studied and $\mathrm{Y}$ are the other $\mathrm{N}$ strains of $\mathrm{X}-\mathrm{Y}$ interactions.

\section{Melanin extraction and quantification}

Melanin was extracted from 2-week-old colonies grown on MEA plates. Mycelia were harvested, dried overnight at $50^{\circ} \mathrm{C}$ and ground to a fine powder. The extraction process was carried out according to the method of (Berthelot et al.2017b). One gram of fungal powder was washed for $5 \mathrm{~min}$ with $30 \mathrm{ml}$ of distilled water, followed by a centrifugation step at 4,000 rpm for $5 \mathrm{~min}$. The supernatant was discarded, and the pellet was resuspended in $6 \mathrm{M} \mathrm{NaOH}$ to a final $\mathrm{pH}$ of 12 . The mixture was sonicated at $40 \mathrm{~Hz}$ for $24 \mathrm{~h}$ at $50^{\circ} \mathrm{C}$ and centrifuged at 4,000 $\mathrm{rpm}$ for $5 \mathrm{~min}$. The alkaline pigmented extract was then acidified to $\mathrm{pH} 2$ with $8 \mathrm{M} \mathrm{HCl}$ to precipitate melanin, followed by a centrifugation step at $10,000 \mathrm{rpm}$ for $20 \mathrm{~min}$. Finally, melanin was washed with chloroform and ethyl acetate. For quantification, melanin was dissolved in $0.1 \mathrm{M} \mathrm{NaOH}$, and the $\mathrm{OD}_{400}$ of the solution was determined. Blanks consisted in 0.1 $\mathrm{M} \mathrm{NaOH}$. A standard curve was obtained with synthetic melanin (M8631, Sigma-Aldrich).

\section{Statistical analyses}

Statistical analyses were performed with R 3.1.3 software ( $\mathrm{R}$ core team 2013). The normality of the data was tested using the Shapiro-Wilk test. Correlations between the measured parameters were evaluated by PCA using the FactoMineR package. The data were then analysed by a one-way non-parametric Kruskal-Wallis test $(\alpha=0.05)$. Pearson correlation coefficients were calculated to determine the relationship between the mycelial melanin content and the effect of the DSEs on the growth of other DSEs, ECM fungi and pathogens. The percentage values were $\arcsin$ transformed before statistical analyses.

\section{Results and discussion}

DSEs live in plant roots and share the same ecological niche with mycorrhizal, endophytic and pathogenic fungi (Bonito et al. 2016). From the perspective of using DSE inocula as promoters of plant growth or as biocontrol agents, it is important to select adequate strains that are either unaffected by or competitive against other root fungal inhabitants. In the context of testing DSE-based inocula for future use in the tree-based phytomanagement of marginal lands, we focused our study on fungal species that were isolated from tree roots sampled in stressed areas (Berthelot et al. 2016). Previous studies investigating the interactions between different fungal strains relied on the measurement of several parameters, such as radial growth, colony diameter or inhibition zone (IZ) between the two strains (Tellenbach et al. 2013; Yan et al. 2015). In the present study, the pertinence of these different parameters was investigated to study the interactions of DSEs co-cultivated with other DSEs, with ECM fungi or with root pathogens (Fig. S1a). The results of the principal component analysis (Fig. S1b) showed that 

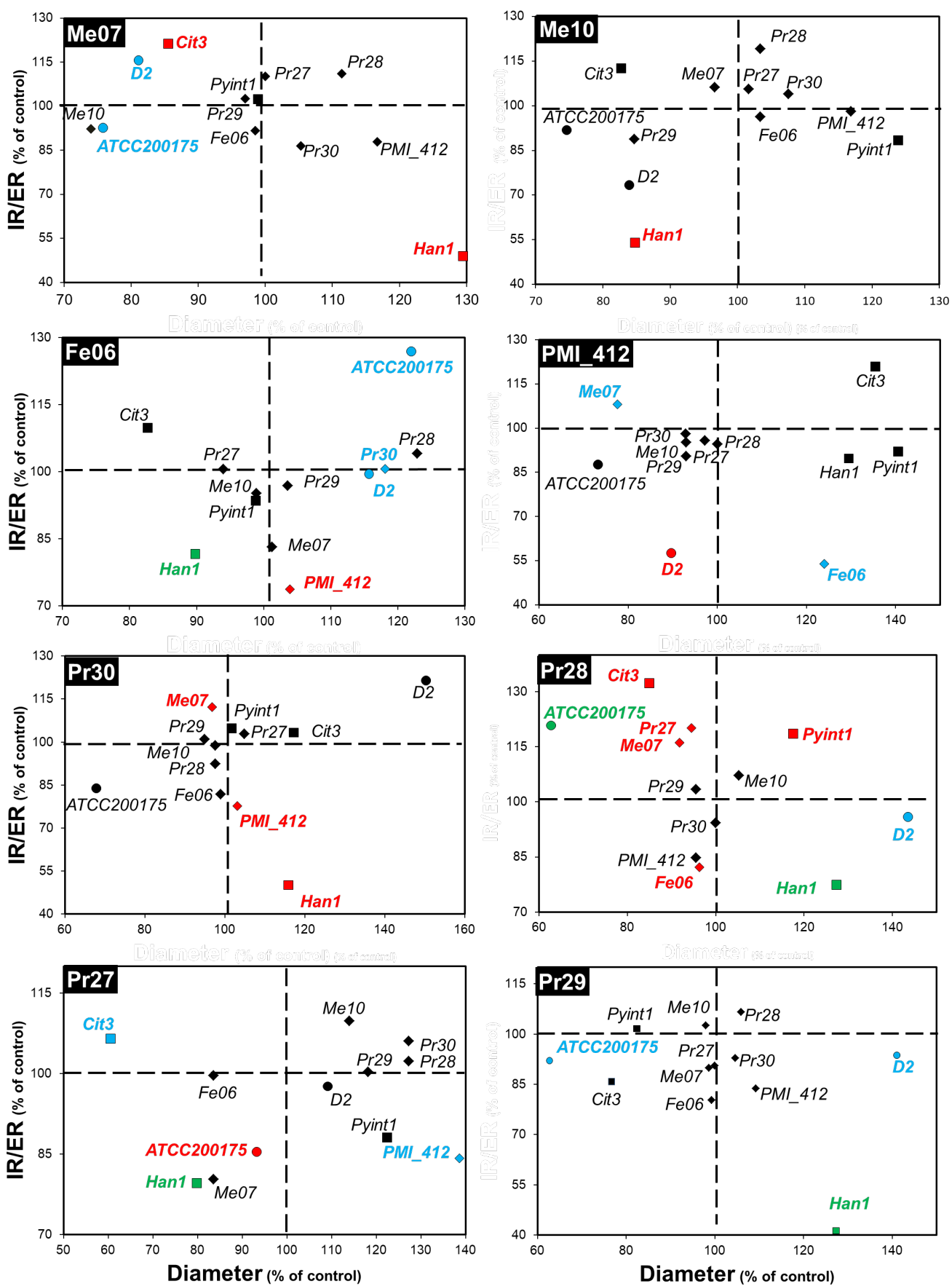

Fig. 1: Outcomes of the interactions between dark septate endopytes (DSEs) and DSEs, ectomycorrhizal fungi or phytopathogens. Fungal co-colutures were performed on MEA plates. The diameter of the colony and the ratio of the internal radius over the external radius (IR/ER) of the colony were the two parameters studied to determine the outcome of the interaction. Each subset of the figure gives the outcomes of the interactions between a given DSE strain (name in the black box on the top left of the subset) and the 12 other strains. Data are the means of triplicates and are expressed as percentages of the control (interaction between two colonies of the same strain). Strains for which the colony diameter, the IR/ER parameter, or both parameters were significantly affected (Kruskal-Wallis, $\mathrm{P}<0.05$ ) by a given DSE strain are given in blue, red and green, respectively. DSEs, ectomycorrhizal fungi and phytopathogens are represented by diamond, circle and square symbols, respectively.

the IZ parameter was negatively correlated with the IR/ER (internal/external radius ratio) parameter, suggesting that the diameter and IR/ER were the best indicators among those that were measured. These parameters were indeed independent of each other, confirming the 
relevance of using both of them to assess the growth response. Therefore, only these two parameters were considered and presented hereafter. The diameter was used as a proxy for strain growth, whereas the IR/ER ratio emphasized the deformation of the fungal colonies. The results of the interactions between the different strains are shown in Fig. 1-3 and Tables S1-S2. In most cases, no physical contact was observed in the interactions (Fig. 4a, b, c, e). In other cases, the colonies were in closer contact (Fig. 4d) or even overlapped (Fig. 4f). A total of 136 combinations of DSEs and DSEs, DSEs and ECM fungi or DSEs and pathogenic strains were tested and the corresponding outcomes of these interactions are described below.

An environmental metabarcoding analysis reported that DSEs are common inhabitants of the roots of trees such as poplar (Bonito et al. 2016; Foulon et al. 2016). Notably, Exophiala and Cadophora were the two most abundant ascomycetous genera represented by DSEs in the rhizosphere of poplar growing on a metal-contaminated site (Foulon et al. 2016). In the present study, the outcomes of the DSE/DSE interactions were very predominantly neutral. Indeed, out of the 56 combinations tested, 52 and 50 neutral interactions were found when the colony diameter (Fig. 1, Table S1) or the IR/ER parameter (Fig. 1, Table S2), respectively, was considered as the marker of the interaction. Three interactions generated synergistic outcomes when considering the diameter parameter (Fig. 1, Table S1). The same number of synergistic outcomes was found for the IR/ER parameter (Fig. 1, Table S2). In contrast, 4 DSE strains significantly inhibited other DSE strains. Notably, the PMI_412 and Pr30 strains of Leptodontidium sp. significantly inhibited the diameter of Leptodontidium sp. Me07 (Fig. 1, Table S1) and the IR/ER ratio of Leptodontidium sp. PMI_412 (Fig. 1, Table S2), respectively. Furthermore, Cadophora sp. Fe06 and $P$. fortinii $\operatorname{Pr} 28$ altered the IR/ER ratio of Leptodontidium sp. PMI_412 and Cadophora sp. Fe06, respectively. Likewise, co-cultures of ascomycetous foliar endophytes such as Cladosporium cladosporioides or Phialophora sp. resulted in neutral to antagonistic interactions (Yan et al. 2015). However, in the present study, the outcome of the DSE/DSE interactions, and the intensity of the growth response appeared to be strain-dependent, at least for Leptodontidium. Further studies should investigate whether this is also the case for interactions between different strains of other DSEs, such as Cadophora or $P$. fortinii. Several studies reported that DSEs produced diffusible and volatile organic compounds that could act as antimicrobial molecules (Berthelot et al. 2016; Terhonen et al. 2016). The few cases of antagonism observed in the present study suggest that these molecules are either not produced in our culture system or do not have antagonistic effects on the other DSE species that were tested. The first hypothesis is unlikely since diffusible molecules were observed around colonies of DSEs and since different putative toxic volatile organic compounds were previously reported to be produced by Cadophora and Leptodontidium strains on the same growth medium (Berthelot et al.2016). However, our data establish the first set of information showing the variability of the responses that occur between DSE species and further reinforce the need to preselect a consortium composed of compatible DSE strains in the setting of tree inoculation.

ECM fungi are also common root inhabitants of most tree species. Therefore, we also aimed to study the impact of DSEs on two well-known ECM species (Paxillus involutus and Hebeloma cylindrosporum) that are widely distributed and are abundant root colonizers in metal-contaminated areas (Op de Beeck er al. 2015). DSEs affected the growth of ECM fungi. Three (of 8) DSE strains significantly reduced the diameter of P. involutus ATCC 200175, whereas Cadophora sp. Fe06 had a synergistic impact on this ECM fungus (Table S1). 

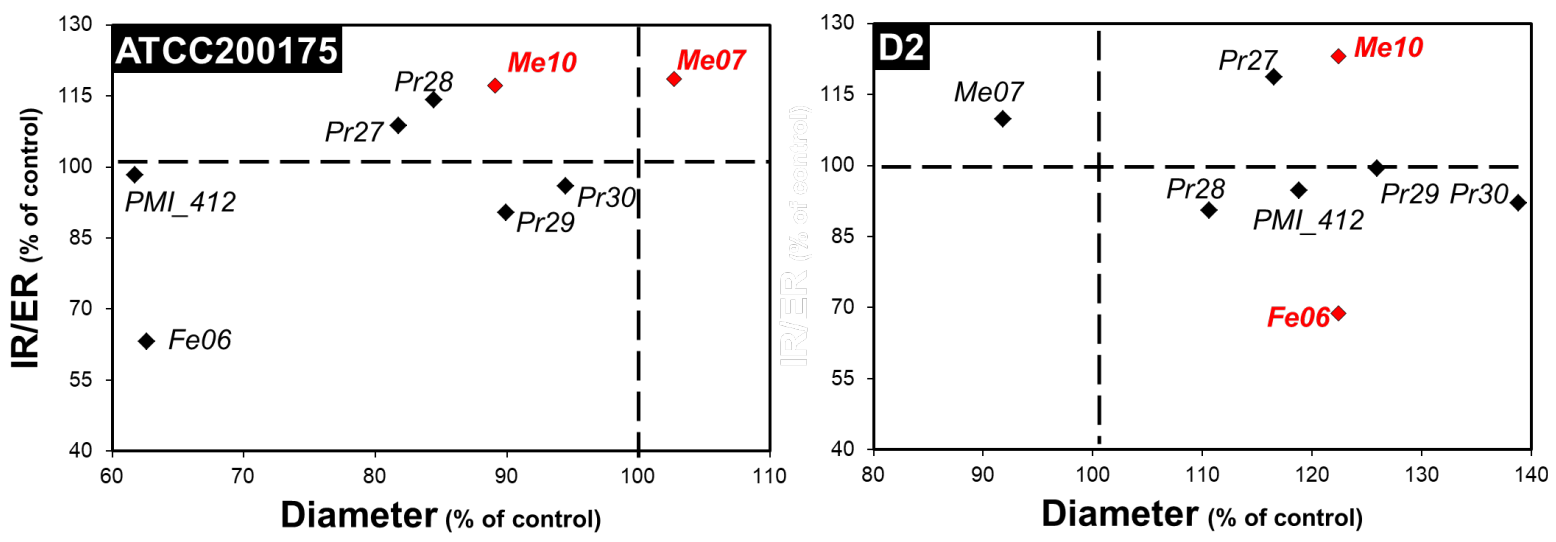

Fig. 2: Outcomes of the interactions between ectomycorrhizal fungi and dark septate endophytes. Fungal co-colutures were performed on MEA plates. The diameter of the colony and the ratio of the internal radius over the external radius (IR/ER) of the colony were the two parameters studied to determine the outcome of the interaction. Each subset of the figure gives the outcomes of the interactions between a given ectomycorrhizal strain (name in the black box on the top left of the subset) and the 8 DSE strains. Data are the means of triplicates and are expressed as percentages of the control (interaction between two colonies of the same strain). Strains for which the colony diameter, the IR/ER parameter, or both parameters were significantly affected (KruskalWallis, $\mathrm{P}<0.05$ ) by a given ectomycorrhizal strain are given in blue, red and green, respectively.

Furthermore, three DSE strains (Cadophora sp. Fe06, P. fortinii $\operatorname{Pr} 28$ and P. mustea Pr29) significantly increased the diameter of $H$. cylindrosporum D2, whereas Leptodontidium sp. $\mathrm{Me} 07$ had the opposite effect (Table S1). When the IR/ER parameter was considered as the interaction marker, two interactions involving Leptodontidium sp.PMI_412 and P. mustea Pr27 generated antagonistic outcomes in co-culture with $H$. cylindrosporum D2 and $P$. involutus ATCC 200175, respectively (Table S2). Conversely, $P$. fortinii Pr28 increased the IR/ER ratio of $P$. involutus ATCC 200175 (Table S2). In addition, we investigated the effect of ECM fungi on DSEs. None of the ECM fungi had an impact on DSEs when the diameter was considered as the interaction marker (Fig. 2, Table S1). For the IR/ER parameter, H. cylindrosporum D2 had a synergistic impact on one of the DSE strains (Leptodontidium sp. Me10) and a negative effect on Cadophora sp. Fe06 (Fig. 2, Table S2). P. involutus ATCC 200175 increased the IR/ER ratio of two Leptodontidium sp. strains (Me10 and Me07) (Fig. 2, Table S2). However, the outcomes of the ECM/DSE interactions were strain-dependent, as exemplified by $P$. involutus ATCC 200175, which increased the growth of only half of the tested Leptodontidium sp. strains (Fig. 2, Table S2). Five studies have reported direct interactions between DSEs and mycorrhizal fungi in vitro and in planta. The DSE Drechslera sp. inhibited the germination of spores of the endomycorrhizal fungus Gigaspora sp. in pairwise assays in vitro (Scervino et al. 2009). The outcome of the dual inoculation of the endomycorrhizal fungus Funneliformis mosseae and Cadophora sp. was neutral for the endomycorrhizal fungus, and the root colonization by Cadophora sp. was slightly reduced (Berthelot et al. 2018). In planta, an antagonistic effect of the ECM fungus Laccaria bicolor on several DSE strains of Phialocephala sp. but not on other strains of the same species was reported, confirming the strain-dependent relation between these two species (Reininger and Sieber 2012) Similarly, the co-inoculation of Norway spruce with $P$. fortinii - Acephala applanata species complex (PAC) and Hebeloma crustuliniforme resulted in a decrease in the PAC biomass compared with that of the control (Reininger and Sieber 2013). However, the plant biomass was higher in the dualinoculation condition than in the single inoculation condition with $H$. crustuliniforme and PAC (Reininger and Sieber 2013). The authors suggested that the plant growth promotion was explained by the ECM-mediated access to plant growth-promoting nutrients by the mineralization of the potting medium by PAC (Reininger and Sieber 2013). Consistent with 
these results, Vohnik et al. 2011 observed that the roots of Rhododendron harboured both ericoid mycorrhiza (ErM) and DSE associations. In this study, a single mycelium simultaneously developed structures corresponding to ErM and DSE associations in roots (Vohník and Albrechtová 2011). The existence of this specific structure could suggest a synergistic lifestyle or a morphological continuum between ErM and DSE.

To test the ability of DSEs to suppress biotic stresses, the putative antifungal capabilities of DSEs were evaluated against the three well-known root pathogens $H$. annosum, $P$. ultimatum and Phytophthora citricola. Two DSE strains, namely, P. mustea Pr27 and Cadophora sp. Fe06, significantly reduced the colony diameter of H. annosum Han1 (Table S1). The same result was observed for the interaction between P. mustea Pr27 and P. citricola Cit3 (Table S1). When the IR/ER parameter was considered as the interaction marker, except for Leptodontidium sp. PMI_412, all DSE strains altered H. annosum Han1 colonies (Table S2). Similar results were reported in the literature, with Cadophora, Phialocephala and Leptodontidium decreasing the growth of other phytopathogenic fungi (Andrade-Linares et al. 2011; Tellenbach et al. 2013; Khastini et al. 2014; Terhonen et al. 2016). However, except for studies on $P$. fortinii, no study examined the interaction between other DSE species and the root pathogens $P$. citricola, $H$. annosum or P. ultimatum (Rasanayagam and Jeffries 1992; Tellenbach and Sieber 2012; Tellenbach et al. 2013; Terhonen et al. 2016). Therefore, the present results further extend our knowledge of DSEs as potential antagonists of other species of pathogenic fungi and oomycetes. Furthermore, in the present study, we tested the impact of pathogenic fungal strains on the growth of DSEs (Fig. 3), a relationship that was usually ignored in previous studies.
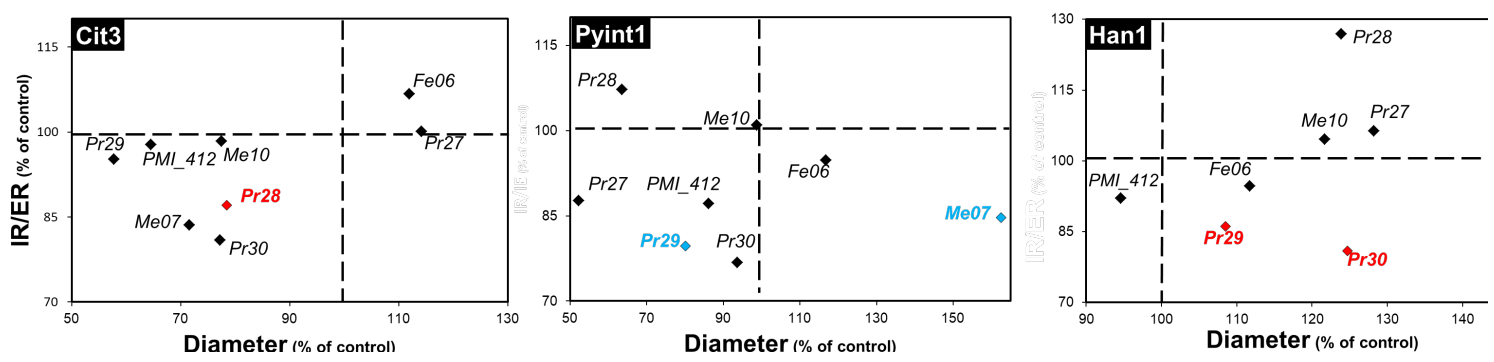

Fig. 3: Outcomes of the interactions between root phytopathogens and dark septate endophytes. Fungal co-colutures were performed on MEA plates. The diameter of the colony and the ratio of the internal radius over the external radius (IR/ER) of the colony were the two parameters studied to determine the outcome of the interaction. Each subset of the figure gives the outcomes of the interactions between a given root phytopathogen (name in the black box on the top left of the subset) and the 8 DSE strains. Data are the means of triplicates and are expressed as percentages of the control (interaction between two colonies of the same strain). Strains for which the colony diameter, the IR/ER parameter, or both parameters were significantly affected (KruskalWallis, $\mathrm{P}<0.05$ ) by a given root phytopathogen are given in blue, red and green, respectively.

Among the 24 combinations tested for the colony diameter as interaction marker, we observed a single outcome of growth inhibition, where $P$. ultimatum Pyint1 affected $P$. mustea Pr29 (Fig. 3, Table S1). Concerning the IR/ER marker, three other negative outcomes were found. P. citricola Cit3 impacted P. fortinii Pr28 and $H$. annosum Han 1 affected both Leptodontidium sp. Pr30 and P. mustea Pr29 (Fig. 3, Table S2). This lack of general inhibition of DSEs might suggest the presence of adaptive mechanisms in DSEs, such as melanin impregnation or defence compounds (Kuo and Alexander 1967; Terhonen et al. 2016). DSEs are enriched in melanin; this is particularly the case for the dark black colonies of Cadophora sp. Fe06 (Berthelot et al. 2017b). To test whether a correlation exists between melanin content 
in the hyphae of the different DSEs and the outcome of the fungal interactions, we first extracted and quantified melanin from the different DSE strains and performed correlation analyses by using the entire data set of fungal interactions. The melanin concentration in the cell walls ranged from 0.89 (Leptodontidium sp. Me07) to $6.67 \mathrm{mg} / \mathrm{g}$ DW (Cadophora sp. Fe06) (Table S3). However, the degree of resistance of the DSE strains towards the three pathogens was not correlated (Pearson test, $P<0.05$ ) with the melanin concentration in their hyphae. Similarly, there was no correlation between the melanin concentration in the hyphae of DSEs and the effect of these fungi on the growth of ECM fungi or other DSEs, as evidenced by the plot reporting the response index for both diameter (RId) and IR/ER ratio (RIr) (Fig. 5).

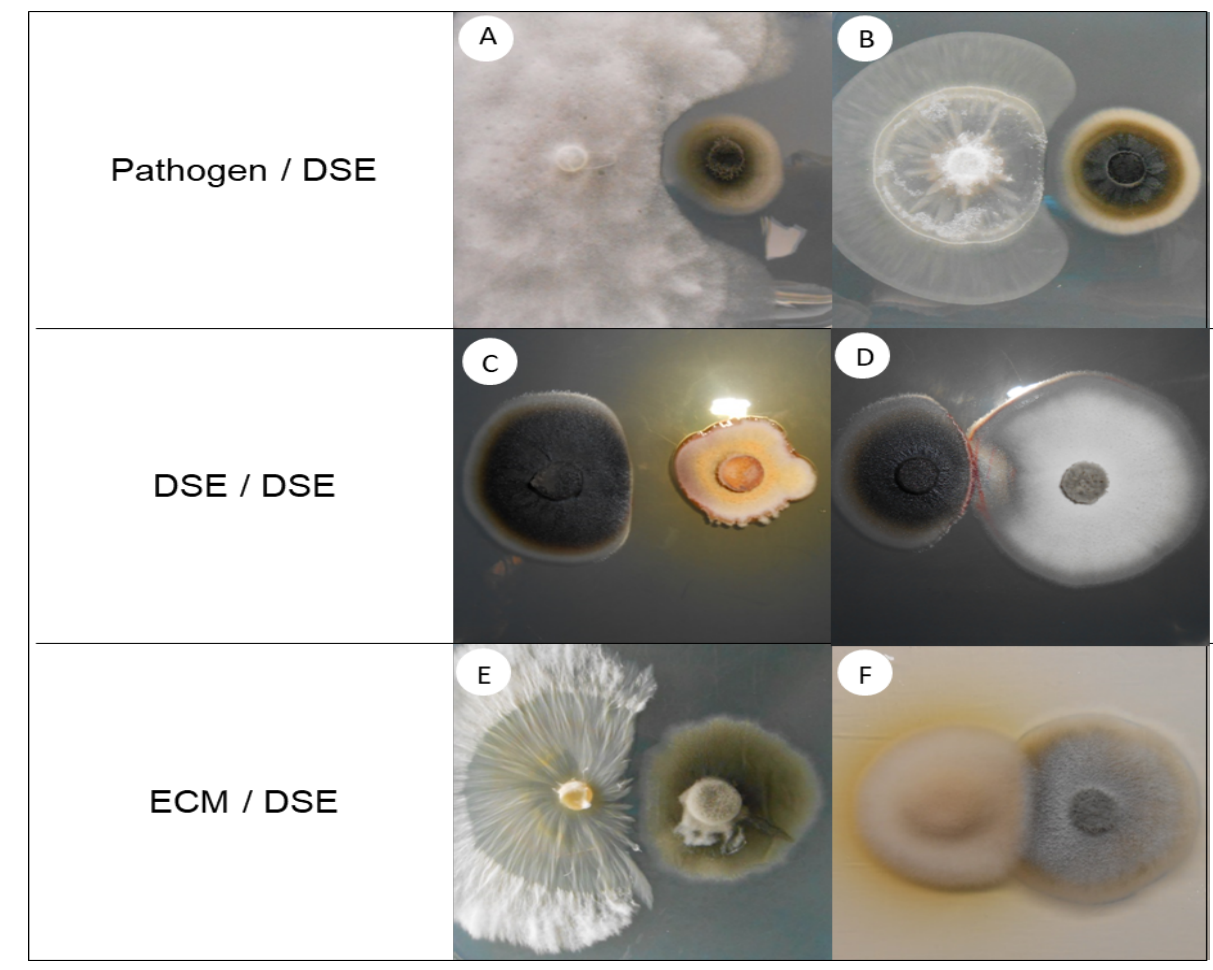

Fig. 4: Dual inoculations among DSE, ectomycorrhizal, and phytopathogenic strains.

The images show the co-cultures (left/right) of (a) Heterobasidion annosum Han 1 / Cadophora sp. Fe06, (b) Phytophthora citricola Cit3 / Phialophora mustea Pr29, (c) Cadophora sp. Fe06 / P. mustea Pr27, (d) Cadophora sp. Fe06 / P. mustea Pr29, (e) Hebeloma cylindrosporum D2 / Leptodontidium sp. PMI_412, and (f) P. involutus ATCC 200175 / Leptodontidium sp. Me10. The images were obtained after two weeks of growth at $24^{\circ} \mathrm{C}$ on MEA medium. A representative plate (out of four) is shown.

In the dual cultures, negative interactions between the strains could result from three different mechanisms: competition for space, competition for nutrients, and antibiosis. Competition for space is the case where the growth of one fungus is stimulated, while the growth of another fungus is inhibited. In addition, competition for nutrients could mediate the interactions between fungi. Several fungi are able to secrete siderophores or acidic compounds to mobilize and acquire nutrients, thus spatially limiting resources for use by other fungi. For instance, Phialocephala sp. were reported to synthesize hydroxamate siderophores (Bartholdy et al. 2001). In addition to siderophore secretion, the release of antibiotic and secondary metabolites could impact fungal growth. For example, Cryptosporiopsis sp. were reported to inhibit the growth of H. annosum through the release of cryptosporin (Terhonen et al. 2016). 


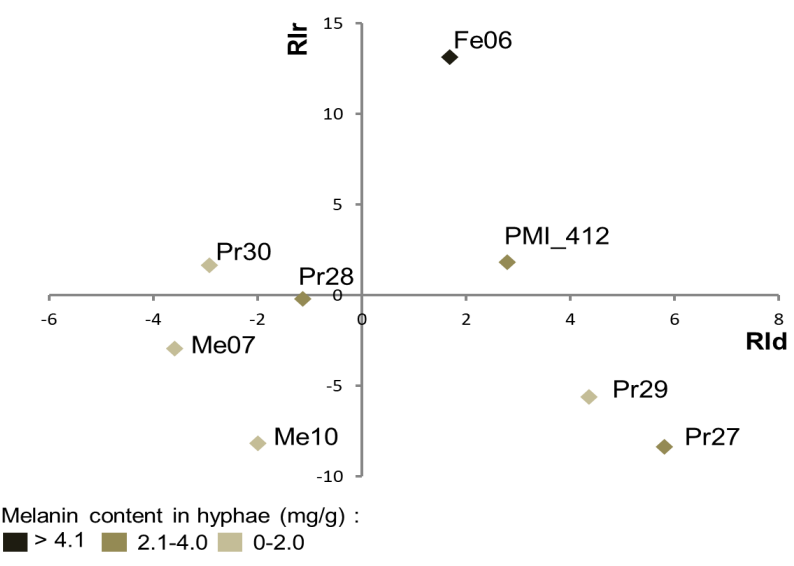

Fig. 5: Two-dimensional plot of the index responses of the DSE strains. DSE strains were plotted on the two dimensional plot using the response index of the diameter (RId; $x$-axis) and the response index of the internal radius / external radius ratio (RIr; $y$-axis). Means used for the calculation of RId and RIr were obtained from independent triplicates.

Likewise, the release of sclerin and sclerotinin A by Phialocephala europea decreased the growth of $P$. citricola (Tellenbach et al. 2013). In our previous work, toxic volatile organic compounds (VOC), such as $\beta$-chamigrene and 1,3-cyclopentadiene 1,3bis, were isolated from the atmosphere of flasks in which Leptodontidium sp. Pr30 grew (Berthelot et al. 2016). This finding suggests, as has been previously suggested for other fungi, that these molecules could be responsible for the inhibitory effects we observed (Kramer and Abraham 2012). Similarly, Fusarium oxysporum was able to produce VOC that highly inhibited the growth of the pathogens Sclerotinia sclerotiorum and Botrytis cinerea (Zhang et al. 2014). In our study, yellow-to-brown halos surrounded the colonies of Cadophora sp. Fe06 and P. mustea Pr27; this result suggests that both strains produce diffusible secondary metabolites that could have inhibitory effects (Fig. 4). However, such compounds remain to be identified and their inhibitory potential tested.

\section{Conclusions}

Our results indicated that the majority of the outcomes of DSE/DSE interactions were neutral and that few antagonistic interactions occurred. The outcomes between ECM fungi and DSEs were more variable than were the interactions between DSEs and organisms of other types and were strain-dependent. Interestingly, we identified several strains acting in synergy with others, as well as strains that could potentially act as biocontrol agents. Moreover, the DSE strains used in the present study exert PGP effects on several plant species and are also metaltolerant (Berthelot et al. 2016, 2017a). Therefore, the present set of data will aid in selecting fungal candidates for consortia of DSE and/or ECM strains that could be used in bioaugmentation strategies for the phytomanagement of marginal lands, which are usually characterized by poor nutrient availability and stressful conditions (metal contamination). However, it is important to consider that this strategy, while essential to the primary step of choosing the most compatible candidates among a large set of strains, does not reflect the complexity of soil/root environments. Therefore, based on the present findings, further investigations will be conducted to evaluate in planta the effect of such consortia on plant growth promotion and biocontrol potential. 


\section{Acknowledgements}

We would like to thank G. Bonito (Michigan State University) and C. Husson (INRA Champenoux) for the gift of the strains PMI_412, Han1, Pyint1 and Cit3 and P. Marques for help with the fungal cultures.

\section{Funding}

This work was supported by the French Environment and Energy Management Agency (PROLIPHYT 1172C0053) and by the LORVER project funded by the Lorraine Region and the European Regional Development Fund (ERDF).

\section{Conflict of interest}

The authors declare that they have no conflict of interest.

\section{References}

Andrade-Linares DR, Grosch R, Franken P, et al (2011) Colonization of roots of cultivated Solanum lycopersicum by dark septate and other ascomycetous endophytes. Mycologia 103:710-721.

Bartholdy B, Berreck M, Haselwandter K (2001) Hydroxamate siderophore synthesis by Phialocephala fortinii, a typical dark septate fungal root endophyte. Biometals 14:33-42.

Berthelot C, Blaudez D, Beguiristain T, et al (2018) Co-inoculation of Lolium perenne with Funneliformis mosseae and the dark septate endophyte Cadophora sp . in a trace elementpolluted soil. Mycorrhiza 28:301-314.

Berthelot C, Blaudez D, Leyval C (2017a) Differential growth promotion of poplar and birch inoculated with three dark septate endophytes in two trace element-contaminated soils. Int J Phytoremediation 19:1118-1125.

Berthelot C, Leyval C, Foulon J, et al (2016) Plant growth promotion, metabolite production and metal tolerance of dark septate endophytes isolated from metal-polluted poplar phytomanagement sites. FEMS Microbiol Ecol 92:fiw144.

Berthelot C, Perrin Y, Leyval C, Blaudez D (2017b) Melanization and ageing are not drawbacks for successful agro-transformation of dark septate endophytes. Fungal Biol 121:652-663.

Bonito G, Hameed K, Ventura R, et al (2016) Isolating a functionally relevant guild of fungi from the root microbiome of Populus. Fungal Ecol 22:35-42.

Caldwell BA, Jumpponen A, Trappe JM (2000) Utilization of major detrital substrates by darkseptate, root endophytes. Mycologia 92:230-232.

Cano C, Bago A (2005) Competition and substrate colonization strategies of three polyxenically grown arbuscular mycorrhizal fungi. Mycologia 97:1201-14.

Della Monica IF, Saparrat MCN, Godeas AM, Scervino JM (2015) The co-existence between DSE and AMF symbionts affects plant $\mathrm{P}$ pools through $\mathrm{P}$ mineralization and solubilization processes. Fungal Ecol 17:10-17.

Foulon J, Zappelini C, Durand A, et al (2016) Environmental metabarcoding reveals contrasting microbial communities at two poplar phytomanagement sites. Sci Total Environ $571: 1230-1240$

Fryar SC, Booth W, Davies J, et al (2005) Evidence of in situ competition between fungi in freshwater. Fungal Divers 59-71 
Jumpponen A, Trappe JM (1998) Dark septate endophytes: A review with special reference to facultative biotrophic root-colonizing fungi. New Phytol 140:295-310.

Khastini RO, Ogawara T, Sato Y, Narisawa K (2014) Control of Fusarium wilt in melon by the fungal endophyte, Cadophora sp. Eur J Plant Pathol 139:333-342.

Kramer R, Abraham WR (2012) Volatile sesquiterpenes from fungi: What are they good for? Phytochem Rev 11:15-37.

Kuo MJ, Alexander M (1967) Inhibition of the lysis of fungi by melanins. J Bacteriol 94:624629

Likar M, Regvar M (2013) Isolates of dark septate endophytes reduce metal uptake and improve physiology of Salix caprea L. Plant Soil 370:593-604.

Mandyam K, Jumpponen A (2005) Seeking the elusive function of the root-colonising dark septate endophytic fungi. Stud Mycol 53:173-189.

Massenssini AM, Bonduki VHA, Tótola MR, et al (2014) Arbuscular mycorrhizal associations and occurrence of dark septate endophytes in the roots of Brazilian weed plants. Mycorrhiza 24:153-9.

Op De Beeck M, Lievens B, Busschaert P, et al (2015) Impact of metal pollution on fungal diversity and community structures. Environ Microbiol 17:2035-47.

Pereira E, Coelho V, Tavares RM, et al (2012) Effect of competitive interactions between ectomycorrhizal and saprotrophic fungi on Castanea sativa performance. Mycorrhiza 22:41-9.

R core team (2013) R: A language and environment for statistical computing. R Foundation for Statistical Computing, Vienna, Austria.

Rasanayagam S, Jeffries P (1992) Production of acid is responsible for antibiosis by some ectomycorrhizal fungi. Mycol Res 96:971-976.

Reininger V, Grunig CR, Sieber TN (2012) Host species and strain combination determine growth reduction of spruce and birch seedlings colonized by root-associated dark septate endophytes. Environ Microbiol 14:1064-1076.

Reininger V, Sieber TN (2013) Mitigation of antagonistic effects on plant growth due to root co-colonization by dark septate endophytes and ectomycorrhiza. Environ Microbiol Rep 5:892-898.

Reininger V, Sieber TN (2012) Mycorrhiza reduces adverse effects of dark septate endophytes (DSE) on growth of conifers. PLoS One 7:e42865.

Scervino JM, Gottlieb a., Silvani V a., et al (2009) Exudates of dark septate endophyte (DSE) modulate the development of the arbuscular mycorrhizal fungus (AMF) Gigaspora rosea . Soil Biol Biochem 41:1753-1756.

Tellenbach C, Sieber TN (2012) Do colonization by dark septate endophytes and elevated temperature affect pathogenicity of oomycetes? FEMS Microbiol Ecol 82:157-168.

Tellenbach C, Sumarah MW, Grünig CR, Miller JD (2013) Inhibition of Phytophthora species by secondary metabolites produced by the dark septate endophyte Phialocephala europaea. Fungal Ecol 6:12-18.

Terhonen E, Sipari N, Asiegbu FO (2016) Inhibition of phytopathogens by fungal root endophytes of Norway spruce. Biol Control 99:53-63.

Vaz ABM, Sampedro I, Siles JA, et al (2012) Arbuscular mycorrhizal colonization of Sorghum vulgare in presence of root endophytic fungi of Myrtus communis. Appl Soil Ecol 61:288294. 
Vohník M, Albrechtová J (2011) The Co-occurrence and morphological continuum between ericoid mycorrhiza and dark septate endophytes in roots of six european rhododendron species. Folia Geobot 46:373-386.

Yan JF, Broughton SJ, Yang SL, Gange AC (2015) Do endophytic fungi grow through their hosts systemically? Fungal Ecol 13:53-59.

Zhang Q, Zhang J, Yang L, et al (2014) Diversity and biocontrol potential of endophytic fungi in Brassica napus. Biol Control 72:98-108.

a

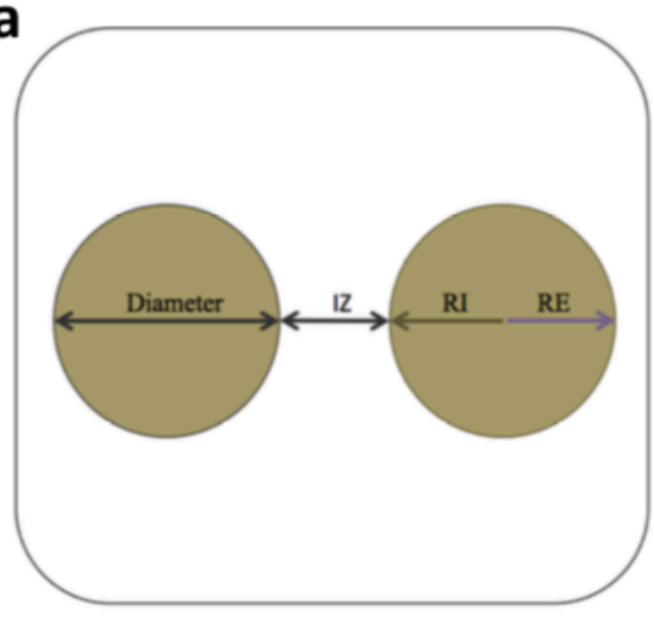

b

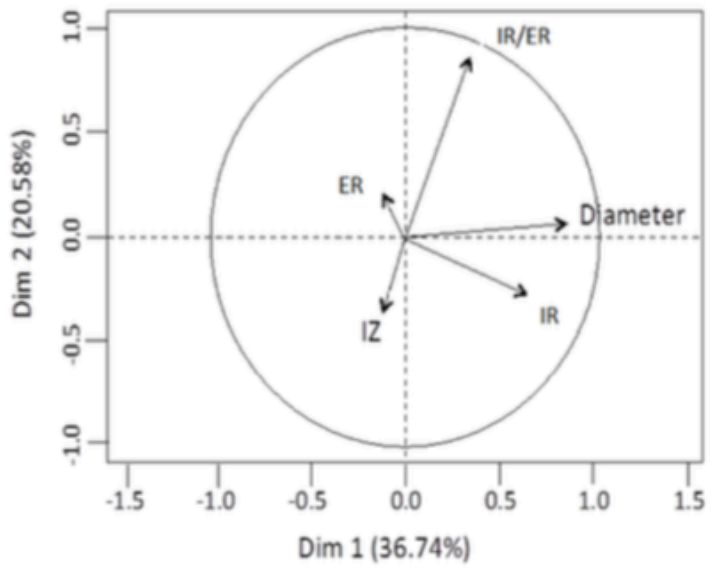

Fig. S1 Experimental design for the study of the interactions of DSEs with DSE, ectomycorrhizal or pathogenic strains.

(a) Experimental design of the pairwise assay and the parameters that were measured. IR and ER are the internal and external radii, respectively; $\mathrm{IZ}$ is the inhibition zone between the two strains. (b) PCA showing the relationship between the parameters that were measured.

\begin{tabular}{|c|c|c|c|c|c|c|c|c|c|c|c|c|c|}
\hline \multirow[b]{3}{*}{ Target strains } & \multicolumn{13}{|c|}{ Fungal strains tested } \\
\hline & \multicolumn{8}{|c|}{ dark septate endophytic } & \multicolumn{2}{|c|}{ Ectomycorrhizal } & \multicolumn{3}{|c|}{ Phytopathogenic } \\
\hline & $\mathrm{Me} 07$ & Me 10 & $\mathrm{P}_{\mathrm{t} 30}$ & PMI_412 & Fe06 & Pr28 & Pr27 & $\mathrm{P} 129$ & ATCC 200175 & D2 & Cit3 & Pyint1 & Han1 \\
\hline Leptodontidium sp. Mc07 & $100.0 \pm 3.4$ & $96.6 \pm 1.7$ & $96.8 \pm 3.4$ & $77.6 \pm 5.7 *$ & $101.2 \pm 2.6$ & $91.8 \pm 13.2$ & $83.6 \pm 8.7$ & $98.7 \pm 13.3$ & $102.7 \pm 12.6$ & $91.8 \pm 3.1$ & $71.6 \pm 21.1$ & $162.5 \pm 20.4^{*}$ & $145.5 \pm 31$. \\
\hline Leptodontidium sp. Me10 & $74.0 \pm 21.9$ & $100.0 \pm 1.4$ & $97.6 \pm 3.5$ & $93.0 \pm 3.8$ & $98.8 \pm 1.5$ & $105.2 \pm 11.3$ & $114.0 \pm 6.2$ & $98.0 \pm 3.8$ & $89.1 \pm 14.0$ & $122.4 \pm 26.7$ & $77.5 \pm 20.0$ & $98.9 \pm 6.7$ & $121.7 \pm 12$. \\
\hline Leptodontidium sp. Pr30 & $105.3 \pm 11.6$ & $107.6 \pm 1.5$ & $100.0 \pm 9.3$ & $92.9 \pm 5.7$ & $118.1 \pm 1.3^{*}$ & $100.0 \pm 11.8$ & $127.3 \pm 17.9$ & $104.6 \pm 12.9$ & $94.5 \pm 1.4$ & $138.8 \pm 20.8$ & $77.3 \pm 22.1$ & $93.8 \pm 5.4$ & $124.7 \pm 19$ \\
\hline Leptodontidium sp. PMI_412 & $116.7 \pm 10.7$ & $116.9 \pm 15.3$ & $103.1 \pm 3.7$ & $100.0 \pm 12.4$ & $103.9 \pm 1.8$ & $95.5 \pm 12.2$ & $138.6 \pm 10.5^{*}$ & $109.2 \pm 10.2$ & $61.8 \pm 17.0$ & $118.8 \pm 29.0$ & $64.6 \pm 37.6$ & $86.3 \pm 12.7$ & $94.6 \pm 1.2$ \\
\hline Cadophora sp. Fe06 & $98.5 \pm 13.4$ & $103.4 \pm 1.3$ & $99.0 \pm 6.7$ & $124.0 \pm 4.3^{*}$ & $100.0 \pm 1.3$ & $96.3 \pm 10.4$ & $83.6 \pm 12.3$ & $99.3 \pm 13.1$ & $62.7 \pm 11.2$ & $122.4 \pm 29.3$ & $112.0 \pm 22.0$ & $116.9 \pm 12.7$ & $111.7 \pm 11$. \\
\hline Phialocephala fortinii $\mathrm{P} 228$ & $111.4 \pm 2.0$ & $103.4 \pm 1.7$ & $97.6 \pm 13.4$ & $100.0 \pm 7.5$ & $122.9 \pm 12.4$ & $100.0 \pm 11.8$ & $127.3 \pm 16.2$ & $105.9 \pm 3.2$ & $84.5 \pm 8.7$ & $110.6 \pm 2.9$ & $78.4 \pm 24.7$ & $63.7 \pm 20.7$ & $123.9 \pm 12$. \\
\hline Phialophora mustea Pr27 & $100.0 \pm 12.3$ & $101.7 \pm 1.9$ & $101.7 \pm 3.5$ & $97.2 \pm 15.6$ & $94.0 \pm 1.3$ & $94.5 \pm 12.2$ & $100.0 \pm 9.1$ & $100.0 \pm 12.7$ & $81.8 \pm 17.6$ & $116.5 \pm 7.8$ & $114.2 \pm 22.1$ & $52.4 \pm 34.6$ & $128.2 \pm 30$ \\
\hline Phialophora mustea Pr29 & $97.0 \pm 11.7$ & $84.7 \pm 18.6$ & $94.9 \pm 5.5$ & $93.0 \pm 7.4$ & $103.6 \pm 1.3$ & $95.5 \pm 11.1$ & $118.2 \pm 8.6$ & $100.0 \pm 8.4$ & $90.0 \pm 1.7$ & $125.9 \pm 31.1$ & $57.8 \pm 31.1$ & $80.1 \pm 5.2^{*}$ & $108.5 \pm 1.3$ \\
\hline Paxillus involutus ATCC 200175 & $75.8 \pm 9.8^{*}$ & $74.6 \pm 15.9$ & $67.8 \pm 23.5$ & $73.2 \pm 15.7$ & $122.0 \pm 2.2^{*}$ & $62.7 \pm 6.1^{*}$ & $93.2 \pm 11.0$ & $62.7 \pm 16.6^{*}$ & $100.0 \pm 13.3$ & nd & $103.6 \pm 11.8$ & $177.3 \pm 8.4^{*}$ & nd \\
\hline Hebeloma cylindrosporum D2 & $81.1 \pm 1.2^{*}$ & $83.9 \pm 11.7$ & $150.3 \pm 30.8$ & $89.7 \pm 12.8$ & $115.7 \pm 1.2^{*}$ & $143.5 \pm 15.4^{*}$ & $109.1 \pm 7.8$ & $141.0 \pm 16.2^{*}$ & nd & $100.0 \pm 5.6$ & $134.1 \pm 21.0$ & $129.4 \pm 18.9$ & nd \\
\hline Phytophthora citricola Cit3 & $85.5 \pm 5.9$ & $82.7 \pm 9.7$ & $117.2 \pm 4.4$ & $135.5 \pm 22.8$ & $82.7 \pm 8.5$ & $85.0 \pm 8.9$ & $60.5 \pm 13.3^{*}$ & $76.7 \pm 16.2$ & $103.1 \pm 3.06$ & $71.6 \pm 26.4$ & $100.0 \pm 9.8$ & nd & nd \\
\hline Pythium intermedium Pyint1 & $98.9 \pm 3.34$ & $123.9 \pm 15.4$ & $104.9 \pm 13.6$ & $140.6 \pm 32.4$ & $98.9 \pm 3.5$ & $117.5 \pm 6.3$ & $122.4 \pm 14.6$ & $82.4 \pm 12.7$ & $80.1 \pm 2.4^{*}$ & $62.2 \pm 31.8$ & nd & $100.0 \pm 12.5$ & nd \\
\hline Heterobasidion annosum Han1 & $129.5 \pm 22.3$ & $84.8 \pm 9.2$ & $115.9 \pm 7.9$ & $129.5 \pm 14.7$ & $89.8 \pm 1.6^{*}$ & $127.3 \pm 4.6^{*}$ & $79.8 \pm 3.8^{*}$ & $127.3 \pm 7.1^{*}$ & nd & nd & nd & nd & $100.0 \pm 3.3$ \\
\hline
\end{tabular}




\begin{tabular}{|c|c|c|c|c|c|c|c|c|c|c|c|c|c|}
\hline \multirow[b]{3}{*}{ Target strains } & \multicolumn{13}{|c|}{ Fungal strains tested } \\
\hline & \multicolumn{8}{|c|}{ dark septate endophytic } & \multicolumn{2}{|c|}{ Ectomycorrhizal } & \multicolumn{3}{|c|}{ Phytopathogenic } \\
\hline & $\mathrm{Me} 07$ & Me10 & $\mathrm{Pr} 30$ & PMI_412 & $\mathrm{Fe} 06$ & $\mathrm{Pr} 28$ & $\mathrm{Pr} 27$ & $\mathrm{Pr} 29$ & ATCC 200175 & D2 & Cit 3 & Pyint1 & Han 1 \\
\hline Leptodontidium sp. Me07 & $100.0 \pm 6.9$ & $106.0 \pm 2.1$ & $112.2 \pm 0.4^{*}$ & $108.1 \pm 5.2$ & $83.1 \pm 12.5$ & $116.1 \pm 1.6^{*}$ & $99.6 \pm 2.7$ & $89.9 \pm 5.1$ & $118.6 \pm 4.9^{*}$ & $109.8 \pm 6.6$ & $83.6 \pm 12.6$ & $84.7 \pm 18.3$ & $89.9 \pm 1.0$ \\
\hline Leptodontidium sp. Mel0 & $92.3 \pm 1.0$ & $100.0 \pm 3.7$ & $98.7 \pm 2.1$ & $95.2 \pm 1.9$ & $93.5 \pm 9.8$ & $107.1 \pm 5.2$ & $109.8 \pm 3.5$ & $102.6 \pm 1.4$ & $117.2 \pm 1.4^{*}$ & $123.1 \pm 10.3^{*}$ & $98.4 \pm 13.3$ & $101.0 \pm 13.6$ & $104.6 \pm 15.0$ \\
\hline Leptodontidium sp. Pr30 & $86.5 \pm 5.0$ & $103.8 \pm 1.5$ & $100.0 \pm 1.5$ & $98.0 \pm 5.2$ & $100.6 \pm 5.0$ & $94.3 \pm 3.2$ & $106.0 \pm 2.2$ & $92.8 \pm 6.7$ & $95.9 \pm 6.1$ & $92.1 \pm 5.1$ & $80.9 \pm 9.7$ & $76.8 \pm 23.7$ & $80.9 \pm 2.13^{*}$ \\
\hline Leptodontidium sp. PMI_412 & $87.9 \pm 4.0$ & $98.0 \pm 0.8$ & $77.7 \pm 1.30^{*}$ & $100 \pm 3.9$ & $73.7 \pm 1.7 *$ & $84.8 \pm 12.6$ & $84.2 \pm 7.8$ & $83.7 \pm 6.9$ & $98.3 \pm 9.3$ & $94.8 \pm 6.9$ & $97.8 \pm 10.7$ & $87.2 \pm 20.0$ & $92.1 \pm 24.0$ \\
\hline Cadophora sp. Fe06 & $91.7 \pm 4.1$ & $96.1 \pm 4.4$ & $81.8 \pm 2.7$ & $53.9 \pm 20.8$ & $100.0 \pm 5.1$ & $82.2 \pm 0.1^{*}$ & $80.3 \pm 11.7$ & $80.3 \pm 14.8$ & $63.1 \pm 27.7$ & $68.8 \pm 1.7^{*}$ & $106.7 \pm 7.3$ & $94.8 \pm 13.4$ & $94.6 \pm 21$ \\
\hline Phialocephala fortinii Pr28 & $111.1 \pm 2.4$ & $119.0 \pm 10.8$ & $92.3 \pm 2.9$ & $94.5 \pm 1.2$ & $104.0 \pm 4.1$ & $100.0 \pm 5.1$ & $102.3 \pm 1.0$ & $106.5 \pm 6.3$ & $114.1 \pm 3.9$ & $90.6 \pm 4.5$ & $87.1 \pm 0.9^{*}$ & $107.2 \pm 16.1$ & $126.9 \pm 24.6$ \\
\hline Phialophora mustea Pr27 & $110.2 \pm 2.8$ & $105.4 \pm 5.9$ & $104.8 \pm 4.1$ & $95.7 \pm 3.8$ & $100.5 \pm 3.8$ & $120.1 \pm 3.9^{*}$ & $100.0 \pm 0.3$ & $90.5 \pm 2.4$ & $108.7 \pm 12.8$ & $118.6 \pm 12.3$ & $100.1 \pm 4.1$ & $87.7 \pm 15.1$ & $106.3 \pm 22.3$ \\
\hline Phialophora mustea $\mathrm{Pr} 29$ & $102.6 \pm 2.8$ & $88.6 \pm 3.7$ & $100.9 \pm 3.0$ & $90.5 \pm 5.5$ & $96.8 \pm 3.5$ & $103.4 \pm 1.5$ & $100.3 \pm 2.7$ & $100.0 \pm 6.8$ & $90.4 \pm 8.0$ & $99.4 \pm 1.7$ & $95.2 \pm 1.5$ & $79.7 \pm 13.9$ & $86.1 \pm 5.3^{*}$ \\
\hline Paxillus involutus ATCC 200175 & $92.6 \pm 7.0$ & $91.8 \pm 10.7$ & $83.9 \pm 5.3$ & $87.6 \pm 2.9$ & $126.9 \pm 16.8$ & $120.8 \pm 10.5$ & $85.4 \pm 3.2^{*}$ & $92.1 \pm 2.3$ & $100.0 \pm 3.0$ & nd & $83.9 \pm 12.7$ & $91.6 \pm 12.5$ & nd \\
\hline Hebeloma cylindrosporum D2 & $115.7 \pm 5.8$ & $73.4 \pm 15.6$ & $121.5 \pm 10.1$ & $57.6 \pm 2.3^{*}$ & $99.5 \pm 0.8$ & $95.9 \pm 6.9$ & $97.6 \pm 7.8$ & $93.7 \pm 3.9$ & nd & $100.0 \pm 3.8$ & $86.9 \pm 8.2$ & $92.8 \pm 15.9$ & nd \\
\hline Phytophthora citricola Cit3 & $121.3 \pm 0.0^{*}$ & $112.6 \pm 4.4$ & $103.3 \pm 1.9$ & $120.9 \pm 13.5$ & $109.8 \pm 5.7$ & $132.3 \pm 1.4^{*}$ & $106.5 \pm 0.0$ & $85.8 \pm 1.3$ & $123.0 \pm 1.4^{*}$ & $116.2 \pm 1.2^{*}$ & $100.0 \pm 5.2$ & nd & nd \\
\hline Pythium intermedium Pyint & $102.4 \pm 0.8$ & $88.4 \pm 4.2$ & $102.8 \pm 0.0$ & $92.1 \pm 1.8 \quad 2 \quad 25$ & $95.1 \pm 0.3$ & $118.5+1.9^{*}$ & $88.1 \pm 4.6$ & $101.6 \pm 0.7$ & $108.3 \pm 0.8$ & $97.4 \pm 14.9$ & nd & $100.0 \pm 5.8$ & nd \\
\hline Heterobasidion annosum Han1 & $48.9 \pm 3.4^{*}$ & $54.0 \pm 6.6^{*}$ & $50.1 \pm 5.1^{*}$ & $89.7 \pm 7.6$ & $81.6 \pm 7.4^{*}$ & $77.4 \pm 5.5^{*}$ & $79.6 \pm 2.4^{*}$ & $41.3 \pm 4.2^{*}$ & nd & nd & nd & nd & $100.0 \pm 5.8$ \\
\hline
\end{tabular}

Table 3: Melanin concentration in the DSE strains

\begin{tabular}{|l|l|}
\hline Strains & Melanin $(\mathrm{mg} / \mathrm{g})$ \\
\hline Leptodontidium sp. $\mathrm{Me} 07$ & $0.89 \pm 0.02$ \\
\hline Leptodontidium sp. $\mathrm{Me} 10$ & $2.01 \pm 0.20$ \\
\hline Leptodontidium sp. Pr30 & $1.51 \pm 0.03$ \\
\hline Leptodontidium sp. PMI_412 & $2.47 \pm 0.05$ \\
\hline Cadophora sp. Fe06 & $6.67 \pm 0.24$ \\
\hline Phialocephala fortinii Pr28 & $3.12 \pm 0.30$ \\
\hline Phialophora mustea Pr27 & $2.30 \pm 0.41$ \\
\hline Phialophora mustea Pr29 & $1.43 \pm 0.22$ \\
\hline Values are means $( \pm \mathrm{SD} ; \mathrm{n}=3)$ & \\
\hline
\end{tabular}

